\title{
Quiste acromioclavicular. Hallazgos en resonancia magnética, a propósito de dos casos clínicos
}

\section{Acromioclavicular Cyst. Findings in Magnetic Resonance, for the Pupose of Two Cases}

\author{
Verónica Gigirey ${ }^{1}$ Laura Valuntas $^{1}$ Corina Bianco ${ }^{1}$ \\ ${ }^{1}$ Centro Uruguayo de Imagenología Molecular, CUDIM, \\ Montevideo, Uruguay \\ Rev Argent Radiol 2018;82:94-97.
}

Estimados editores,

El quiste sinovial acromioclavicular, entidad poco frecuente, es reportado por primera vez por Craig, y col. ${ }^{1}$ en el año 1984. Desde esa fecha y hasta el año 2009, se reportaron 41 casos en la literatura. ${ }^{2}$ Es más frecuente en pacientes de edad avanzada, se presenta como una tumoración adherida a planos profundos, generalmente indolora, sin signos fluxivos. Las técnicas de imagen permiten confirmar el diagnóstico y descartar diferenciales, fundamentalmente con lesiones malignas.

Presentamos dos casos a los que se les realizó una resonancia magnética (RM) con diagnóstico probable de quiste acromioclavicular. El primero, se trataba de una paciente de 77 años del sexo femenino, con una tumoración de hombro derecho, indolora, de un año de evolución. La radiografía mostró una tumoración de partes blandas, superior a la articulación acromioclavicular (AC), bien delimitada, sin lesiones óseas (-Fig. 1). La RM mostró una formación quística, superior y en contacto con la articulación AC, sin realce con el contraste, de $86 \mathrm{~mm}$ de diámetro, bien delimitada, hiperintensa en secuencias ponderadas en T1 (alto contenido proteico) e hiperintensa en densidad protónica con saturación grasa (DPFS). Ademas, se observó una rotura completa del tendón del supraespinoso (—Fig. 2).

El segundo caso correspondió a un paciente de 70 años del sexo masculino, con una tumoración pétrea de hombro derecho, de dos meses de evolución, con aumento brusco de su tamaño. La RM mostró una tumoración quística, hipointensa en secuencias ponderadas en T1 e hiperintensa en DPFS, en estrecha relación con la articulación AC, de $60 \mathrm{~mm}$ de diámetro y rotura completa del tendón supraespinoso ( - Fig. 3 ).

Si bien no se logró demostrar claramente la comunicación entre el quiste y la articulación glenohumeral $(\mathrm{GH})$ por las

received

February 25, 2017

accepted

October 5, 2017

published online

March 23, 2018
Address for correspondence Verónica Gigirey, Av. Ricaldoni 2010, código postal 11600. Teléfono. 24803238, Centro Uruguayo de Imagenología Molecular, CUDIM, Montevideo, Uruguay (e-mail: veronica.gigirey@cudim.org).

imágenes de RM (lo cual muchas veces no es fácil de poner en evidencia ya que no es un estudio dinámico), las alteraciones descriptas fueron interpretadas como sugestivas de quiste acromioclavicular, lo cual fue confirmado quirúrgicamente.

Hiller, y col. ${ }^{2}$ clasifican al quiste acromioclavicular en dos tipos. En el primero, no existe rotura del tendón del manguito rotador y el quiste no comunica con la articulación GH. Su etiología incluye cambios degenerativos secundarios a

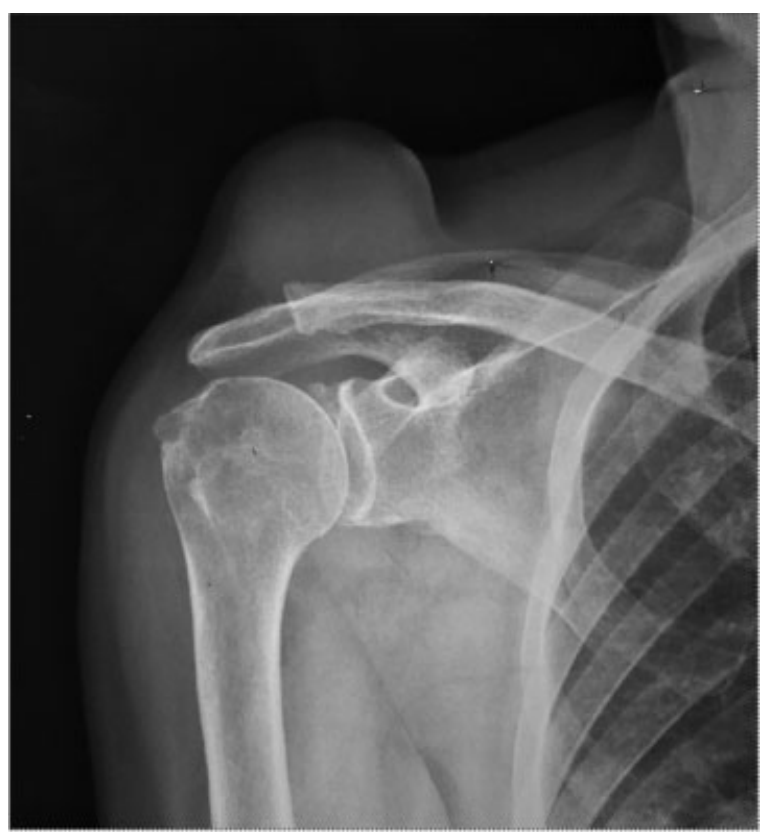

Fig. 1 Radiografía de frente en rotación externa de hombro derecho. Opacidad nodular en partes blandas por encima de la articulación acromioclavicular (AC).
Copyright $\odot$ 2019, Sociedad Argentina de Radiología. Publicado por Thieme Revinter Publicações Ltda., Rio de Janeiro, Brazil. Todos los derechos reservados.
License terms

$10.1055 / \mathrm{s}-0038-1639492$

ISSN 1852-9992.

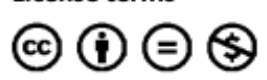



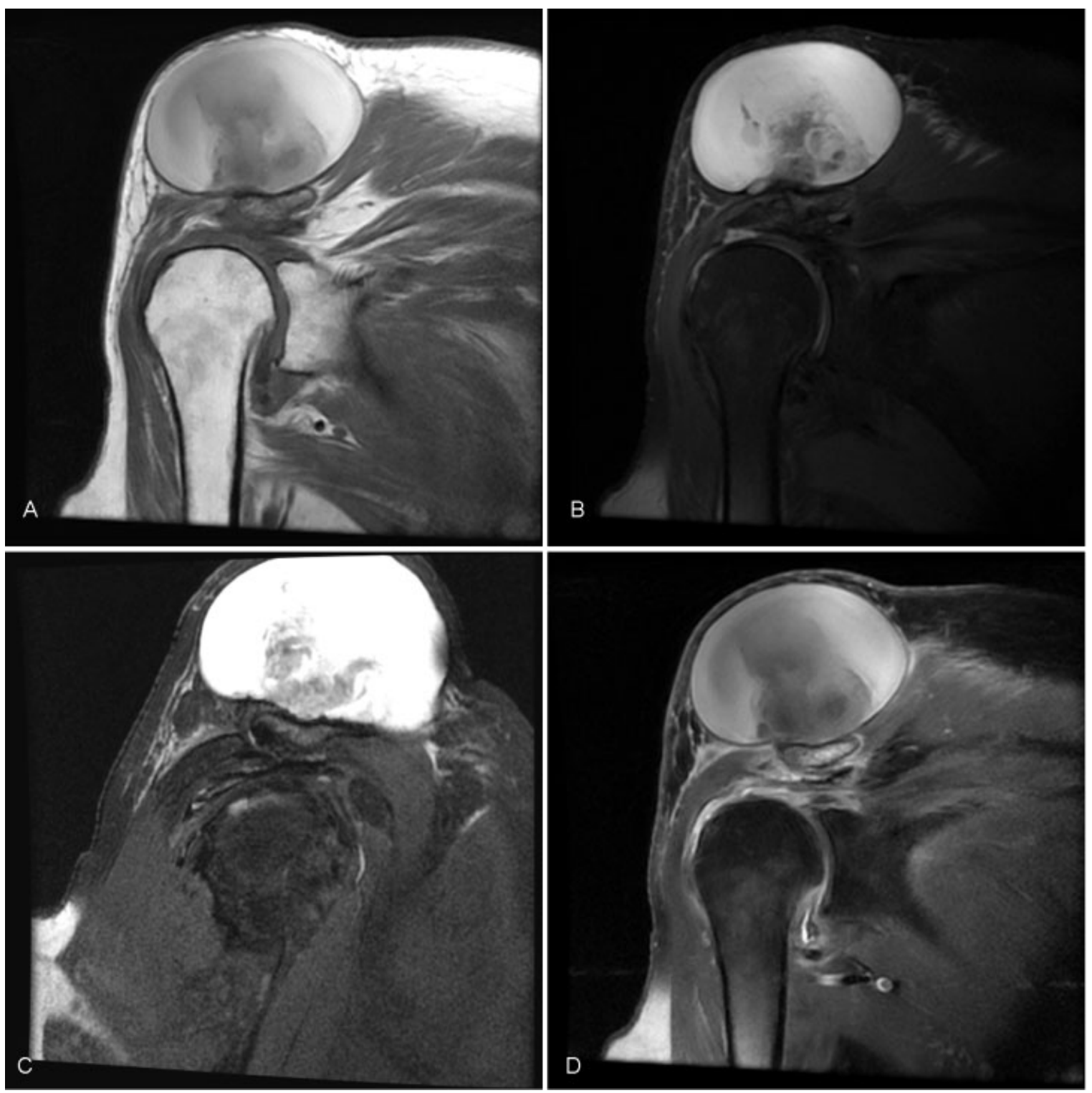

Fig. 2 Resonancia magnética (RM) de hombro derecho, (A) coronal en secuencias ponderadas en T1, (B) coronal DPFS (densidad protónica con supresión grasa), (C) sagital DPFS, (D) secuencia en plano coronal ponderada en T1 supresión grasa con contraste. Quiste acromioclavicular con elevada concentración proteica, hiperintenso en secuencias ponderadas en T1 y T2, sin realce poscontraste. Rotura de espesor completo del tendón del supraespinoso.

traumatismos, infección, enfermedad metabólica o sobreuso. Los cambios degenerativos determinan irritación de la sinovial, con sobreproducción de líquido, lo cual lleva a la formación del quiste. En el segundo existe rotura completa del tendón del manguito rotador. En este caso el ascenso de la cabeza humeral en los movimientos de elevación del brazo produce una fricción repetida sobre la cápsula articular inferior AC, la que se debilita determinando finalmente una solución de continuidad. Eso permite el pasaje del líquido desde la articulación GH hacia la bursa subacromiosubdeltoidea y a la articulación AC. Como consecuencia de ello, la cápsula articular $\mathrm{AC}$ se distiende, determinando un aumento de la presión en la cavidad articular, lo que produce evaginación de la capsula generalmente en sentido cefálico. ${ }^{2,3}$

En cuanto a las pruebas de imagen, la radiografía muestra un aumento de partes blandas en la región superior a la articulación AC. Puede mostrar cambios degenerativos en esa articulación con o sin signos indirectos de rotura del manguito rotador (ascenso de la cabeza humeral, disminución del espacio subacromial y esclerosis glenohumeral). ${ }^{4}$ La artrografía convencional, al ser una técnica dinámica, permite demostrar el pasaje del contraste desde la articulación GH a la bursa y a través de la articulación AC hacia el quiste ("signo del géiser"). ${ }^{4}$

La ecografía y la RM permiten demostrar la naturaleza quística de la lesión, realizar el diagnóstico diferencial con otras entidades benignas como lipomas, mixoma yuxtaarticular, adenopatía o con procesos sólidos malignos. $^{4}$ En la ecografía, se presentan como masas anecoicas, compresibles, sin vascularización con Doppler color y puede poner en evidencia la comunicación con la articulación acromioclavicular. ${ }^{4}$ La RM es útil para detectar la naturaleza quística de la lesión por su hipointensidad en secuencias ponderadas en $\mathrm{T} 1$, hiperintensidad en 

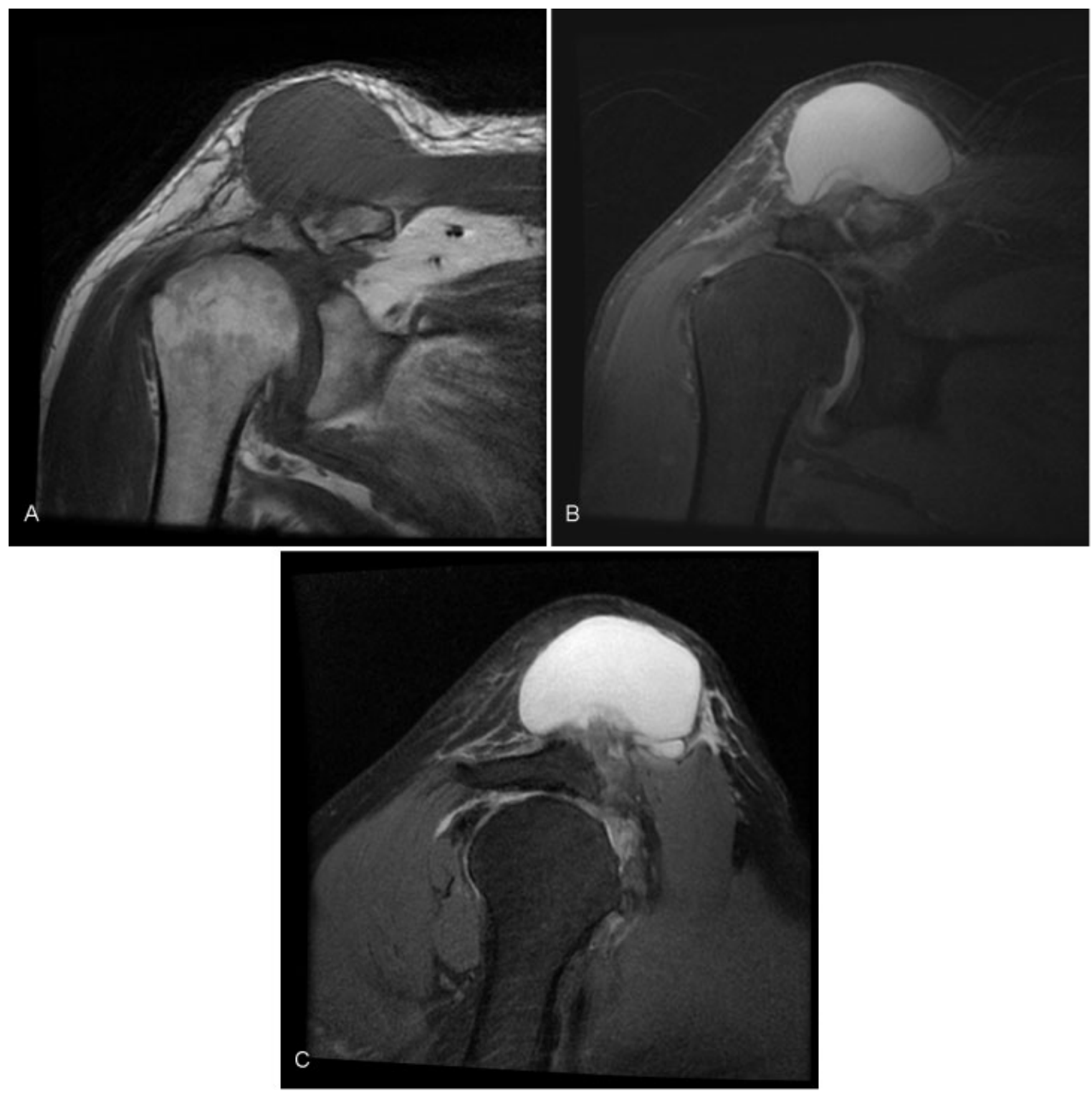

Fig. 3 RM de hombro derecho, (A) coronal en secuencias ponderadas en T1, (B) coronal DPFS y (C) sagital DPFS. Formación quística con estrecho contacto con la articulación acromioclavicular, hipointensa en secuencias ponderadas en T1, hiperintensa en DPFS, asociada a rotura completa del tendón supraespinoso.

secuencias ponderadas en $\mathrm{T} 2 \mathrm{y}$ ausencia de realce con el contraste. $^{4,5}$

Las opciones terapéuticas del quiste acromioclavicular incluyen el tratamiento conservador o quirúrgico, lo cual va a depender de la edad, sintomatología y estado funcional del hombro. En pacientes de edad avanzada, con desgarro masivo del manguito rotador, sin o con escasos síntomas y buena función del hombro, se recomienda un tratamiento conservador. La punción aspirativa tiene un alto indice de recidiva, por lo cual no se recomienda. El tratamiento quirúrgico es de elección en pacientes con dolor crónico y compromiso funcional. En el quiste del primer tipo, se recomienda la resección quirúrgica del mismo con extracción del extremo distal de la clavícula y de la bursa subacromial. En el quiste del segundo tipo, el tratamiento es controvertido; además de la resección quirúrgica del quiste y la reparación del manguito rotador, incluye la resección de la clavícula distal, lavado y desbridamiento artroscópico; artrodesis acromioclavicular; hemiartroplastia humeral, artroplastia total o artroplastia total invertida de hombro. ${ }^{6-8}$

\section{Confidencialidad de los datos}

Los autores declaran que han seguido los protocolos de su centro de trabajo sobre la publicación de datos de pacientes y que todos los pacientes incluidos en el estudio han recibido información suficiente y han dado su consentimiento informado por escrito para participar en dicho estudio.

\section{Conflicto de Intereses}

Los autores del trabajo declaran no tener ningún conflicto de intereses. 


\section{Bibliografía}

1 Craig EV. The acromioclavicular joint cyst. An unusual presentation of a rotator cuff tear. Clin Orthop Relat Res 1986; (202):189-192

2 Hiller AD, Miller JD, Zeller JL. Acromioclavicular joint cyst formation. Clin Anat 2010;23(02):145-152

3 Tshering Vogel DW, Steinbach LS, Hertel R, Bernhard J, Stauffer E, Anderson SE. Acromioclavicular joint cyst: nine cases of a pseudotumor of the shoulder. Skeletal Radiol 2005;34(05):260-265

4 Khor AY, Wong SB. Clinics in diagnostic imaging (151). Acromioclavicular joint geyser sign with chronic full-thickness supraspinatus tendon (SST) tear. Singapore Med J 2014;55(02): 53-56, quiz 57
5 Cooper HJ, Milillo R, Klein DA, DiFelice GS. The MRI geyser sign: acromioclavicular joint cysts in the setting of a chronic rotator cuff tear. Am J Orthop 2011;40(06):E118-E121

6 Murena L, D'angelo F, Falvo DA, Vulcano E. Surgical treatment of an aseptic fistulized acromioclavicular joint cyst: a case report and review of the literature. Cases J 2009;2:8388

7 Shaarani SR, Mullett H. Reverse Total Shoulder Replacement with Minimal ACJ Excision Arthroplasty for Management of Massive ACJ Cyst - A Case Report. Open Orthop J 2014; 8:298-301

8 Marques P, Alpoim B, Rodrigues M, Sá P, Lima F, Rodrigues A. Artroplastia total invertida do ombro em doente com quisto acrómio-clavicular. Rev Port Ortop Traumatol 2013;21:71-76 